\title{
Calidad y preparación en cápsula endoscópica
}

\author{
Gerardo Blanco-Velasco* \\ Hospital de Especialidades, Centro Médico Nacional Siglo XXI, Instituto Mexicano del Seguro Social, Ciudad de México, México
}

Debido a la existencia de pocas guías que hablen de calidad en cápsula endoscópica (CE), la guía de la Sociedad Europea de Endoscopia Gastrointestinal (ESGE) $)^{1}$ publicada en el 2019 continúa siendo el parámetro en el que se basan la mayoría de los trabajos relacionados con calidad y preparación. En los ESGE Days 2020 se aceptaron 4 trabajos relacionados con calidad de la CE y 2 de preparación de la CE, mientras que en la DDW 2020 se aceptaron 5 de calidad y 1 de preparación. En el siguiente artículo se comentaran los trabajos más significativos.

En el apego a las guías se aceptó en los ESGE Days 2020 un trabajo realizado por Triantafyllou, et al. ${ }^{2}$, en el que encuestaron a 217 endoscopistas. El 91\% de estos mencionaron que se adhirieron a las guías ESGE aunque solo el $73.3 \%$ pertenecían a la ESGE. Estos endoscopistas dieron información verbal o escrita a los pacientes a los que les realizaron el estudio de CE que incluía: las indicaciones de la CE en el $84 \%$, las contraindicaciones en el $70.8 \%$, el riesgo de retención en 93.6\% y la necesidad de preparación para el procedimiento en el $78 \%$. Para la preparación previa a la colocación de la CE, el $62.3 \%$ utilizó dieta con líquidos claros, $85.4 \%$ laxantes, $73.2 \%$ simeticona y $56 \%$ procinéticos. Tan solo el $20 \%$ de estos endoscopistas midió la adecuada preparación del intestino delgado (ID) durante el estudio de CE. En cuanto a las indicaciones, la guía ESGE de calidad recomienda utilizar la CE en hemorragia, anemia por deficiencia de hierro, enfermedad de Crohn, tumores, síndromes polipósicos, anormalidades radiológicas y enfermedad celiaca; y no lo recomienda en dolor abdominal, diarrea y malabsorción por los bajos resultados de la CE. Sin embargo, existen otras patologías donde la CE puede ser útil y en un futuro se podrían convertir en una nueva indicación. Una de ellas es la enfermedad injerto contra huésped (EICH), en los ESGE Days 2020, se acepto un trabajo realizado en el Hospital de Especialidades del Centro Médico Nacional Siglo $X X I$, en el que se comparó la utilidad de la CE contra la histopatología que es el estándar de oro en $\mathrm{EICH}$. La cápsula mostró tener una sensibilidad del $88 \%$, especificidad del $75 \%$, valor predictivo positivo (VPP) del $94 \%$ y valor predictivo negativo (VPN) del $60 \% 3$.

En cuanto a la selección del paciente se presentó un estudio que incluyó 55 pacientes con dispositivos cardíacos (DC) (42 pacientes con marcapasos y 13 con dispositivos cardíacos implantables), los cuales son una contraindicación relativa para la colocación de CE debido a la interferencia que puede existir entre estos dispositivos y la CE. A estos pacientes se les colocó una $C E$ observando que no existió disfunción de los DC durante el estudio. Pero al comparar estos estudios de CE con 55 pacientes sin DC en los que también se colocó, se observó que los pacientes con DC tenían mayor cantidad de imágenes perdidas (17 vs. 5; $p=0.004)$. Estas imágenes perdidas tenían una duración promedio de 13.4 minutos y la mayoría eran en colon (9 pacientes). Se concluye que la CE es un estudio seguro en pacientes con DC, pero que existe el riesgo de que se pierdan imágenes de la $\mathrm{CE}^{4}$. 
En cuanto a la visualización completa del ID, la guía ESGE recomienda que esta debe ser igual o mayor al $80 \%$ de las CE colocadas, pero idealmente mayor 0 igual al 95\% de los estudios de CE. Un trabajo aceptado en la DDW 2020 propuso realizar estudios de CE bajo inmersión para mejorar la tasa de visualización completa y la calidad de las imágenes. Esta consiste en dar $500 \mathrm{ml}$ de agua en el momento en que la cápsula pasa a duodeno utilizando la imagen en tiempo real de la grabadora y posteriormente $500 \mathrm{ml}$ cada hora hasta que la cápsula llegara a colon o se terminara la batería. Este estudio incluyó a 59 pacientes en el grupo de inmersión y a 58 en el grupo control. En los resultados se mostró que el grupo de inmersión tuvo incremento significativo en la visualización completa del ID (92.19\% vs. $76.32 \%$ ), además de que la calidad de las imágenes fueron mejores en el tercio proximal y medio $(p=0.0007$ y 0.002 , respectivamente) $)^{5}$.

Referente a las complicaciones, se aceptó en la DDW 2020, una revisión sistemática de enero del 2000 a marzo del 2019 en el que se incluyó a 402 estudios que incluían 108,079 procedimientos. Se observó que la retención se producía en el $0.73 \%(0.59 \%-0.89 \%)$, problemas para la deglución de la CE en $0.75 \%(0.43 \%$ $1.13 \%)$, problemas técnicos en el $0.94 \%(0.65 \%-1.28 \%)$ y complicaciones totales en $0.67 \%(0.32 \%-1.10 \%)$ de todos los estudios de CE. No se presentaron complicaciones por aspiración. La edad avanzada se asoció con visualización incompleta del ID $(p=0.007)$ y se observó que las tasas de retención y visualización incompleta disminuyeron con el paso de los años $(p=0.0006 \text { y } p<0.0001 \text {, respectivamente })^{6}$.

Las guías ESGE recomiendan el utilizar cápsula Patency (CP) en aquellos pacientes con alto riesgo de estenosis en ID (enfermedad de Crohn confirmada, síntomas de obstrucción, uso prologado de antiinflamatorios no esteroideos o radiación pélvica). Un estudio demostró la superioridad de la CP en sensibilidad, especificidad, VPP y VPN (83\%, 100\%, 100\% y 96\%; respectivamente) contra la enterografía por resonancia magnética (ERM) $67 \%, 92 \%, 67 \%$ y $92 \%$; respectivamente) para predecir la retención de la $\mathrm{CE}$. Además demostró que la concordancia de la CP es mayor que la ERM para detectar estenosis comparado con la CE y enteroscopia (0.88 vs. 0.58$)^{7}$.

La preparación es una de las cuestiones en las que existe más controversia en el estudio de CE. La revisión técnica realizada por la $\mathrm{ESGE}^{8}$ recomienda la utilización de 2 litros de polietilen-glicol (PEG) para mejorar la visualización de las imágenes de la CE. La guía de calidad en CE de la ESGE', sugiere utilizar escalas de medición de limpieza en especial la de Brotz o la de Park. La escala de Brotz es probablemente las más utilizada y puede ser cuantitativa (del 0 al 10) y/o cualitativa (excelente, buena, regular y mala) ${ }^{9}$ En Ios ESGE Days 2020, se aceptó un trabajo que incluía a 110 pacientes a los que se les colocó la CE, en 58 pacientes la preparación consistió en líquidos claros, mientras que 52 se prepararon con 2 litros de PEG. Los pacientes del grupo de PEG tuvieron una mejor preparación utilizando la escala cuantitativa de Brotz ( $8.13 \pm 1.56$ vs. $7.47 \pm 1.97 ; p=0.05)$, mientras que no hubo diferencia significativa utilizando la escala cualitativa de Brotz $(p=0.67)$, ni en la capacidad diagnóstica $(p=0.7)^{10}$.

La guía de calidad de la ESGE ${ }^{1}$, también recomienda la utilización de 80 a $200 \mathrm{mg}$ de simeticona para mejorar la calidad de la visualización de la mucosa. Se aceptaron dos trabajos relacionados con simeticona. El primero fue aceptado en la DDW 2020 e incluyó a 159 pacientes divididos en dos grupos. Uno de los grupos era de alto volumen y se les daba a los pacientes $1125 \mathrm{mg}$ de simeticona en $750 \mathrm{ml}$ de agua previo al estudio de CE. El segundo grupo era de control, y se preparaban con $300 \mathrm{mg}$ de simeticona en $200 \mathrm{ml}$ de agua. No se observaron diferencias estadísticas entre ambos grupos, ni en la limpieza del ID, ni en la capacidad diagnóstica ${ }^{11}$. El segundo trabajo relacionado con simeticona fue en Ios ESGE Days 2020. En este se incluyeron a 96 pacientes, de los cuales 32 se preparaban con $100 \mathrm{mg}$ de simeticona y a 64 pacientes no se les daba simeticona. Comparando ambos grupos, no se encontró diferencia estadísticamente significativa en la limpieza ni en la capacidad diagnóstica. Sin embargo, el grupo de simeticona tuvo mayor visualización completa del ID $(100 \text { vs. } 92 \% ; p=0.03)^{12}$.

Con los estudios aceptados en ambos congresos se puede concluir que la EICH puede ser una nueva indicación de CE y que esta se puede utilizar de forma segura en pacientes con dispositivos cardíacos, aunque existe el riesgo de que se pierdan imágenes. También que la CE bajo inmersión mejora la calidad de las imágenes en los dos tercios proximales del ID, que la retención y la visualización incompleta disminuyen con el paso de los años y que la CP es superior a la ERM para predecir retención de la CE. En cuanto a la preparación, concluimos que la simeticona no mejora la limpieza ni la capacidad diagnóstica y que la preparación con PEG (2L) es superior a la preparación con líquidos claros. 


\section{Conflicto de intereses}

Los autores declaran no tener conflicto de intereses alguno.

\section{Financiamiento}

Los autores no recibieron patrocinio para llevar a cabo este artículo.

\section{Bibliografía}

1. Spada C, McNamara D, Despot EJ, et al. Performance measures for small-bowel endoscopy: a European Society of Gastrointestinal Endoscopy (ESGE) quality improvement initiative. Endoscopy 2019;51:574-598.

2. Triantafyllou $K$, Lazaridis LD, Beaumont $H$, et al. ESGE guidelines for small-bowel capsule endoscopy: from recommendations to practice. Endoscopy 2020;52:S158-S159.

3. Blanco-Velasco G, Palos-Cuellar R, Domínguez-García MR, et al. Accuracy of capsule endoscopy in the diagnosis of gastrointestinal graft-versus-host-disease. Endoscopy 2020; 52:S269.
4. Condon AS, Lenhart A, Grewal J, et al. Small bowel capsule endoscopy and cardiac implantable electronic devices: what is the real risk of interference? Gastrointest Endosc 2020;91:AB610.

5. Zeng $X$, Jiang $S, Y e ~ L$, et al. The value of diving method for capsule endoscopy in examination of small intestinal disease: a prospective randomized trial. Gastrointest Endosc 2020;91:AB96.

6. Wang YC, Pan J, Liu YW, et al. Complications of video capsule endoscopy over the past two decades: a systematic review and meta-analysis. Gastrointest Endosc 2020;91:AB608.

7. Blanco-Velasco G, Ramos-García J, Solórzano-Pineda O, et al. Efficacy and safety of patency capsule compared to magnetic resonance enterography for prediction of capsule endoscopy retention in patients with Crohn disease. Gastrointest Endosc 2020;91:AB622.

8. Rondonotti E, Spada C, Adler S, et al. Small-bowel capsule endoscopy and device-assisted enteroscopy for diagnosis and treatment of smaII-bowel disorders: European Society of Gastrointestinal Endoscopy (ESGE) Technical Review. Endoscopy 2018;50:423-446.

9. Brotz C, Nandi N, Conn M, et al. A Validation Study of 3 Grading Systems to Evaluate Small-Bowel Cleansing for Wireless Capsule Endoscopy: A Quantitative Index, a Qualitative Evaluation, and an Overall Adequacy Assessment Gastrointest Endosc 2009;69:262-270.

10. Gomes AC, Ponte A, Pinho R, et al. Preparation protocols before capsule endoscopy. Endoscopy 2020;52:S248-S249.

11. Sey M., Yan B, McDonald C, et al. A randomized double blind clinical trial of high volume simethicone to improve visualization during small intestinal capsule endoscopy. Gastrointest Endosc 2020;91:AB607.

12. Douglas A, Sihag S, Semenov S, et al. Simethicone clears the way for capsules. Endoscopy 2020;52:S270. 